LA-UR- $94-3149$

Conf- $940187-2$

Los Alamos National Laboratory is operated by the University of California for the United States Department of Energy under contract W-7405-ENG-36.

TITLE: EQUILIBRIUM AND SHOT NOISE IN MESOSCOPIC SYSTEMS

AUTHOR(S): Thierry Martin, T-Division/CNLS

SUвMITTED TO XXIXth Rencontres de Moriond "Coulomb and Interference

Effects in Small Electronic Sturctures," January 22-29, 1994, J.

Tran Van Tan, Editor (Editions Frontieres, Paris, France)

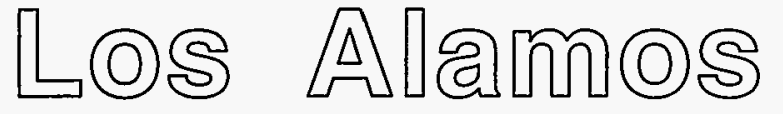

FORM NO. 836 R4

ST. NO. 2629 5/81
Los Alamos National Laboratory Los Alamos, New Mexico 87545

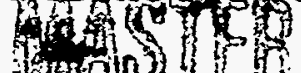

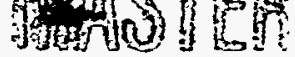




\section{DISCLAIMER}

Portions of this document may be illegible in electronic image products. Images are produced from the best available original document. 


\title{
EQUILIBRIUM AND SHOT NOISE IN MESOSCOPIC SYSTEMS
}

\author{
Thierry Martin, Theory Division, CNLS, Los Alamos National Laboratory
}

\section{INTRODUCTION}

Within the last decade, there has been a resurgence of interest in the study of noise in Mesoscopic devices, both experimentally and theoretically ${ }^{1-27)}$. Noise in solid state devices can have different origins: there is $1 / f$ noise ${ }^{28}$, which is believed to arise from fluctuations in the resistance of the sample due to the motion of impurities ${ }^{29}$. On top of this contribution is a frequency independent component associated with the stochastic nature of electron transport, which will be the focus of this paper.

If the sample considered is small enough that dephasing and inelastic effects can be neglected, equilibrium (thermal) and excess noise can be completely described in terms of the elastic scattering properties of the sample. As mentioned above, noise arises as a consequence of random processes governing the transport of electrons. Here, there are two sources randomness: first, electrons incident on the sample occupy a given energy state with a probability given by the Fermi-Dirac distribution function. Secondly, electrons can be transmitted across the sample or reflected in the same reservoir where they came from with a probability given by the quantum mechanical transmission/ reflection coefficients. Equilibrium noise refers to the case where no bias voltage is applied between the leads connected to the sample, where thermal agitation alone allows the electrons close to the Fermi level to tunnel trough the sample ${ }^{23}$. In general, equilibrium noise is related to the conductance of the sample via the Johnson-Nyquist formula ${ }^{30}$. In the presence of a bias, in the classical regime, we expect to recover the full shot noise $\left\langle\Delta^{2} I\right\rangle=2 I \Delta \mu$ as was observed a long time ago in vacuum diodes ${ }^{31}$. In the Mesoscopic regime, however, excess noise is reduced below the shot noise level, as will be explained below.

If the sample is to be described quantum mechanically, a calculation of the noise should include the Pauli principle: an electron which is successfully transmitted cannot occupy the same state as another electron incident from the opposite side, which is reflected by the potential barrier. The importance of the statistics of the charge carriers is somehow a novelty in Mesoscopic physics. After all, many experiments in Mesoscopic physics can have a direct analog if we interchange the carriers with bosonic particles. The conductance steps experiment ${ }^{32}$ ) which 
shows the transverse quantization of the electron wave function is an example: this experiment has been successfully completed with photons ${ }^{33)}$. The measurement of universal conductance fluctuations in Mesoscopic wires and rings ${ }^{34,35)}$ also has an analog when one shines a laser in white paint and studies the speckle pattern generated in this way ${ }^{36)}$. The Aharonov-Bohm effect was successfully observed for electrons in disordered rings ${ }^{35}$ ): nothing tells us that we could not repeat the experiment for bosonic particles. In contrast to these examples, a noise measurement makes a distinction between electrons and photons if we look and the correlations between two detectors collecting the carriers.

Several approaches have been proposed to calculate noise. Some are quasi-classical, as they rely on the concept of trajectory. Others use a formulation of non equilibrium thermodynamics which is based on the concept of reservoirs, introduced for the conductance formula ${ }^{37)}$. The reservoir picture can be cast in a systematic way to calculate the current current correlations in time for a multi-terminal conductor" 38 ). Below we will refer to this treatment as the "poor man's" Keldysh ${ }^{39)}$ approach. In contrast to this point of view, we will introduce a more intuitive picture $^{4,14,15)}$, where the current passing through the device is a superposition of pulses, or electrons wave packets, which can be transmitted or reflected.

\section{HISTORY}

I will start with an historical overview: the list which appears below is by no means exhaustive, and I apologize for the contributions which have been left out.

In 1984, came the first printed suspicion that excess noise could be reduced below shot noise by quantum mechanical effects ${ }^{1}$. It was observed that by varying the voltage bias applied on a point contact, coherent stimulated phonon emission processes reduce the excess noise contribution in a point contact. A theoretical confirmation of this experiment followed soon after $^{2}$. The noise is reduced by a factor $d / l$, where $d$ is the diameter of the point contact and $l$ is the mean free path associated with the phonons $(d<<l)$. The second derivative of the noise with respect to the bias can be related to the electron phonon interaction function. Noise in normal metal-metal and metal-superconducting junctions was analyzed using a semiclassical Keldysh approach ${ }^{3}$, with an identification of the thermal and non-equilibrium contributions. For a junction between normal metal and superconductor, an additional contribution to noise comes from Andreev reflection processes ${ }^{40}$ ). In 1989, the wave packet approach was introduced to calculate thermal equilibrium noise ${ }^{4)}$ : the calculation was done for a sample connected to two leads each carrying one channel. Around the same time, the noise in the presence of a bias was calculated using the "poor man's" Keldysh approach"), for an adiabatic point contact (no mixing between the different transverse modes). Momentum noise was investigated in single and multiple barrier geometries ${ }^{6}$. Two experiments measuring low frequency noise were performed by the Princeton group. The first one considered a point contact ${ }^{7)}$, as in the conductance step experiment ${ }^{32}$ and measured the noise as a function of the gate voltage of the contact, and the second used a resonant tunneling geometry ${ }^{8} i$ The noise was measured 
at a frequency of the order of $10 \mathrm{KHz}$, which is sufficiently low that the $1 / f$ contribution to the noise still has to be subtracted out to determine the white noise contribution we are interested in. Both experiments showed qualitatively that in the case of poor transmission, the noise level corresponds to full shot noise, while highly transmissive samples give a level which is lower than shot noise. In the point contact experiment, a new channel is opened up by varying the gate voltage, while for the resonant tunneling experiment, the transmission of electrons depends on the proximity of the incident energy to the quasi level in the center region. The result of Ref. 5 was then generalized to samples connected to an arbitrary number of leads ${ }^{9}$, where the noise was expressed directly in terms of the general S-matrix describing the scattering properties of the sample, in analogy with the earlier work on multi-probe current measurements ${ }^{38}$ ). Noise was measured in the quantum Hall regime ${ }^{10,11)}$ : noise arises when electrons are backscattered from one edge of the sample to the other. Noise plateaus were detected $^{10}$ ) at the same location as the resistance plateaus associated with the (fractional) quantum Hall regime. A semiclassical approach to the calculation of noise which used phase space trajectories ${ }^{12)}$ showed once again that the noise vanishes for ideal transmission. The wave packet approach introduced for thermal equilibrium in Ref. 4 was extended to treat the non- equilibrium situation ${ }^{14}$, and then generalized to the case of multi-channel, multi-terminal samples to discuss noise correlations ${ }^{15}$ ). The probability distribution for charge transfer in a Mesoscopic sample was found to be close to a binomial distribution ${ }^{16)}$, suggesting that the current is indeed emitted in "bursts". It was pointed out" ${ }^{17)}$ that in a four terminal geometry, noise correlations can yield flux sensitive information. Recently, an interference effect similar to that of Ref. 17 was pointed out for a two terminal geometry ${ }^{26)}$, where there the sample is bent in the form of a loop and an alternating flux passes through the loop. Frequency dependent noise has been studied by several authors ${ }^{3,5,18)}$. Noise was proposed ${ }^{19)}$ as a diagnostic of tunneling mechanism: in a resonant tunneling geometry, the noise power exhibits sharp variations where a current measurement fails to give similar information. The effect of dephasing and dissipation on the quantum noise has been analyzed ${ }^{20}$ ) with the conclusion that dephasing alone is not sufficient to recover the macroscopic regime. Electron-electron interactions were included for a geometry where two ideal leads are connected by a central interacting region ${ }^{22)}$. Electron correlations where also considered recently in the fractional quantum Hall regime ${ }^{27)}$. Sample averaging was introduced ${ }^{13)}$ to study the suppression of shot noise in diffusive conductors, and later on in a study of the Mesoscopic fluctuations in the shot noise power of metals ${ }^{25}$ ) Shot noise was computed in the semiclassical regime for a metallic conductor ${ }^{23)}$, and it was found to be reduced by a factor equal to the ratio of the mean free path divided by the sample length.

\section{THE WAVE PACKET APPROACH}

We consider first a one dimensional sample connected to a source and a drain. The quantity we wish to calculate is the time correlation in the current:

$$
\left.C(t)=\frac{1}{T} \int d t^{\prime}<I\left(t+t^{\prime}\right) I\left(t^{\prime}\right)\right\rangle
$$


The spectral density of noise $W(\omega)$ is related to the above quantity by a simple Fourier transform. The measurement frequencies which we consider here are low enough compared to the inverse of the time associated with the transfer of an electron from source to drain ${ }^{41)}$ and allow to neglect the self inductance of the sample. Using the Fourier representation for the current, this yields:

$$
W(\omega)=\lim _{T \rightarrow \infty}\left(\frac{2}{T}<|I(\omega)|^{2}>\right)
$$

where the angular backets denote some kind of average over electrons occupation factors. In the "poor man's" Keldysh approach, this is equivalent to taking a thermodynamic average with a generalized grand canonical weight $\exp \left(-\beta\left(H-\sum_{i} \mu_{i} N_{i}\right)\right)$, where $N_{i}$ is the number operator counting the scattering states incident from a given lead $i$. The nonequilibrium situation is met when all chemical potentials $\mu_{i}$ are not equal. A justification of this choice of thermal weight has been provided in Ref. 42. If we assume the different reservoirs to be disconnected at a time $t=-\infty$, and adiabatically turn on the coupling between the reservoirs to its full value at $t=0$, the Lipman-Schwinger equation "transforms" the creation/anihilation operators for each reservoir into operators describing scattering states. As long as the difference between chemical potentials is sufficiently small so that we can neglect the charge pileup in the sample connecting the reservoirs, this approach provides useful answers, and constitutes a systematic way to calculate current current correlations from the S-matrix.

The wave packet approach views the current passing across the sample as a superposition of clocked pulses ${ }^{4,14,14)}$ :

$$
I(t)=\sum_{n} j(t-n \tau) g_{n}
$$

In this expression $j(t)$ is the current associated with a given pulse and $g_{n}$ is an occupation factor which takes a value 1 if an electron has been transferred from the left hand side to the right hand side of the sample, -1 if the electron was transferred from right to left, and 0 when no electron is transferred at all. The quantum mechanics necessary to calculate the noise is hidden in $g_{n}$. The wave packets representing the electrons are separated in time, but can overlap in space. This view of electron transport is somewhat supported in Ref. 16, where the probability distribution of charge transfer is found to be close to a binomial Bernouilli distribution with an average number of attempts in the time $t$ equal to $2 \mathrm{eV} / \mathrm{ht}$. An example of wave packet construction can be obtained if we consider states limited to a small energy interval $\Delta E$ :

$$
\psi^{(n)}(z, t)=\int_{E-\Delta E}^{E+\Delta E} \frac{d E^{\prime}}{\Delta E}\left[\frac{1}{2 \pi} \frac{d k}{d E^{\prime}}\right]^{1 / 2} e^{i k\left(E^{\prime}\right) z-i E^{\prime}(t+n \tau) / \hbar},
$$

by choosing $\tau=h / \Delta E$, we insure that successive pulses are orthogonal to each other. With the above definitions, the calculation of the noise contribution coming from the frequency interval $[\nu, \nu+\delta \nu]$ and the energy interval $[E-\Delta E / 2, E+\Delta E / 2]$ reduces to the calculation of the fluctuation in the occupation factors: 


$$
<\Delta I^{2}>_{\delta}=\frac{2 \delta \nu \Delta E e^{2}}{\pi \hbar}<g^{2}-<g>^{2}>\text {. }
$$

where we have dropped the index $n$ in $g_{n}$ because all pulses contribute to the noise in the same fashion. Also, note that we have subtracted the average current in order to describe the effect of the applied bias on the sample. The calculation of the spectral density of noise is thus directly related to the statistics of the current pulses.

To obtain the correlator $\left\langle g^{2}-\langle g\rangle^{2}\right\rangle$, we consider all possible pulse histories: first consider the case where two electrons are incident on the sample from opposite sides. In this situation $g=0$, because there will be no current if both electrons are reflected or transmitted, and the situation where one electron is reflected and the other is transmitted is forbidden by the Pauli principle; two electrons (with the same spin) cannot occupy the same wave packet state. Secondly, there is the straightforward situation where both incident states are empty, with $g=0$. The other possibilities where $g=0$ follow if an electron is reflected from one side, when no electron was incident from the other side. In fact the only possibilities where a current passes trough the sample are when an electron incident from the right (left) is transmitted while no electron was present on the other side, giving the result $g=1(g=-1)$ with respective weight $f_{1}\left(1-f_{2}\right) T\left(f_{2}\left(1-f_{1}\right) T\right) . f_{1}\left(f_{2}\right)$ is the Fermi-Dirac distribution associated with the left (right) reservoir, and $T$ is the transmission probability. We therefore obtain:

$$
<g^{2}-<g>^{2}>=T\left(f_{1}+f_{2}-2 f_{1} f_{2}\right)-\left(f_{1}-f_{2}\right)^{2} T^{2}
$$

Summing now over all energy intervals, we thus obtain the total excess noise:

$$
\begin{aligned}
<\Delta I^{2}>_{\delta}= & \frac{4 e^{2} \delta \nu}{\pi \hbar} \int d E T(E) f_{2}\left(1-f_{1}\right) \\
& +\frac{2 e^{2} \delta \nu}{\pi \hbar} \int d E T(E)\left(f_{1}-f_{2}\right)\left[1-T(E)\left(f_{1}-f_{2}\right)\right] .
\end{aligned}
$$

In the absence of bias or at high temperatures $\left(\left|\mu_{1}-\mu_{2}\right|<<k_{B} \Theta\right)$, the first term on the right hand side dominates, and we recover the Johnson Nyquist ${ }^{30}$ ) formula for thermal equilibrium noise $e^{4)}$

$$
<\Delta I^{2}>_{\delta}=\frac{4 e^{2} \delta \nu}{\pi \hbar} k_{B} \Theta T .
$$

In the opposite limit, $\left|\mu_{1}-\mu_{2}\right|>>k_{B} \Theta$, we get a contribution which looks like shot noise, except that it is reduced by a factor $1-T$ :

$$
<\Delta I^{2}>_{\delta \nu}=2 e \delta \nu<I>(1-T) .
$$

The reduced shot noise is also called partition noise. In the limit of poor transmission, $T<<1$, and we recover the full shot noise. For highly transmissive channels, $T \sim 1$, and we can think of the deduction of shot noise as being the noise contribution associated with the poor transmission of holes across the sample. Because of the Pauli principle, a full steam of electrons which is transmitted with unit probability does not contribute to noise. Note that this is the effect seen qualitatively in the Princeton experiment ${ }^{10,11}$. In the intermediate regime $\left|\mu_{1}-\mu_{2}\right| \simeq k_{B} \Theta$, there is no clear separation between the thermal and the reduced shot noise contribution. 


\section{GENERALIZATION TO MULTI-CHANNEL CASE}

We now turn to the more complex situation where each lead connected to the sample has several channels. Our concern in this case is the role of channel mixing: a receiving channel on the right hand side collects electrons from all incoming channels transmitted from the left and all reflected channels on the right. We therefore expect that wave packets from these different incoming channel will interfere with each other. To avoid the issue of interference between channels and treat the system as a superposition of one dimensional contributions, we must find a wave packet representation where the mixing between channels is absent.

This representation is obtained by using a decomposition of the $\mathrm{S}$ - matrix describing the sample ${ }^{43)}$. Let us assume for simplicity that both leads have the same number of channels $M$. The S-matrix is then a block matrix containing four $M$ by $M$ submatrices describing the reflection from the right (left) hand side, $\mathbf{s}_{22}\left(\mathbf{s}_{11}\right)$, and the transmission from left to right (right to left), $\mathbf{s}_{12}\left(\mathbf{s}_{21}\right)$ :

$$
S=\left(\begin{array}{l}
s_{11} s_{12} \\
s_{21} s_{22}
\end{array}\right) .
$$

From the unitarity of the S-matrix, which follows from current conservation, it is possible to write the submatrices in terms of two diagonal matrices and four unitary matrices, as long as $s_{11}^{\dagger}$ has no eigenvalue equal to 0 or 1 :

$$
\begin{aligned}
& \mathbf{s}_{11}=-i \mathbf{V}_{1} \mathbf{R}^{1 / 2} U_{1}^{\dagger} \\
& \mathbf{s}_{12}=\mathbf{V}_{1} \mathbf{T}^{1 / 2} U_{2}^{\dagger} \\
& \mathbf{s}_{21}=\mathbf{V}_{2} \mathbf{T}^{1 / 2} \mathbf{U}_{1}^{\dagger} \\
& \mathbf{s}_{22}=-i \mathbf{V}_{2} \mathbf{R}^{1 / 2} U_{2}^{\dagger}
\end{aligned}
$$

where $\mathbf{R}^{1 / 2}$ and $\mathbf{T}^{1 / 2}$ are real diagonal matrices with diagonal elements $R_{i}^{1 / 2}$ and $T_{i}^{1 / 2}$ such that their square $R_{i}$ and $T_{i}$ are the eigenvalues of the matrices $s_{11}^{\dagger} s_{11}$ and $s_{21}^{\dagger} s_{21} . T_{i}$ and $R_{i}$ satisfy the identity $T_{i}+R_{i}=1$, and $\mathrm{U}_{1}, \mathrm{U}_{2}, \mathrm{~V}_{1}, \mathrm{~V}_{2}$ are unitary transformations. This decomposition of the S-matrix has been implicitly pointed out ${ }^{44)}$ in the context of random matrix theory, where the transfer matrix is in general the quantity of interest.

Using the unitary transformations, we can now choose a new basis of incoming and outgoing states on the left and the right side of the sample: $U_{1}\left(V_{1}\right)$ is the unitary transformation used to represent the incoming (outgoing) states on the left side, while $\mathbf{U}_{2}\left(\mathbf{V}_{2}\right)$ is the unitary transformation used to represent the incoming (outgoing) states on the right side of the sample. The effective S-matrix thus obtained in this new basis is a block matrix of four diagonal matrices: 


$$
\tilde{\mathbf{S}}=\left(\begin{array}{cccccc}
-i R_{1}^{1 / 2} & \ldots & 0 & T_{1}^{1 / 2} & \ldots & 0 \\
\vdots & \ddots & \vdots & \vdots & \ddots & \vdots \\
0 & \ldots & -i R_{M}^{1 / 2} & 0 & \ldots & T_{M}^{1 / 2} \\
T_{1}^{1 / 2} & \ldots & 0 & -i R_{1}^{1 / 2} & \ldots & 0 \\
\vdots & \ddots & \vdots & \vdots & \ddots & \vdots \\
0 & \ldots & T_{M}^{1 / 2} & 0 & \ldots & -i R_{M}^{1 / 2}
\end{array}\right)
$$

Note that $\tilde{\mathbf{S}}$ corresponds to a situation where no mixing between channels occurs, effectively a superposition of one dimensional $(2 \times 2)$ S-matrices which are totally decoupled (see Fig. 1).

Fig. 1

In fact this is precisely the form which was assumed by Lesovik ${ }^{5}$ ) for the adiabatic point contact. The absence of correlations between the different incoming and outgoing wave packets allows us to write the noise as a superposition of $M$ one dimensional contributions:

$$
\begin{aligned}
<\Delta I^{2}>_{\delta}= & \frac{4 e^{2} \delta \nu}{\pi \hbar} \int d E \sum_{i=1}^{N} T_{i}(E) f_{2}\left(1-f_{1}\right) \\
& +\frac{2 e^{2} \delta \nu}{\pi \hbar} \int d E \sum_{i=1}^{N} T_{i}(E)\left(f_{1}-f_{2}\right)\left[1-T_{i}(E)\left(f_{1}-f_{2}\right) .\right.
\end{aligned}
$$

This expression can be cast in terms of the block elements of the initial S-matrix using the properties of the trace, $\sum_{i} T_{i}^{n}=\operatorname{Tr}\left[\left(\mathbf{s}_{21}^{\dagger} \mathrm{s}_{21}\right)^{n}\right]$.

The above decomposition was based on the fact that none of the $T_{i}^{\text {'s }}$ are 0 or 1 . We can escape these pathological cases by arguing that we can choose $T_{i} \neq 0,1$ but arbitrarily close to these values. But this is not even necessary; a decomposition similar to Eq. (11d) can still be found such that the effective S-matrix still has the four diagonal block structure. The case where the number of channels on each side is different can be handled in a similar manner: we can simply add a number of passive channels which are totally reflected on the side with fewer channels in order to come back to the case where there are $M$ channels on both sides. Because 
they correspond to states which are totally reflected, passive channels do not contribute to noise.

We make the following remarks: First, a number of experiments have studied the effect of backscattering in the Integer Quantum hall regime in a confined geometry where the electrons propagate along the edges the sample, a reminiscence of the classical skipping orbits ${ }^{45)}$. In a typical experiment on a GaAs heterostructure, the backscattering of electrons from one edge to the other can be controlled by varying the voltage on a metallic gate placed across the Hall bar $^{46)}$. As the gate voltage depletes the electrons underneath, the innermost edge state is the first to experience backscattering, while the other ones are fully transmitted. In the theorist mind, assuming that there are no irregularities in the lateral confinement potential, and in the absence of impurities, neighboring edge states do not $\operatorname{mix}^{47)}$, and the S-matrix associated with this situation has precisely the four diagonal structure discussed above. That is to say, there is some grain of physical reality in the decomposition of the S-matrix. Secondly, we note that in the presence of time reversal symmetry, we get additional constraints on the unitary transformations, the transformation used to describe the incident states on a given side of the sample must coincide with the transformation associated with the outgoing states on the same side: $\mathrm{U}_{1}=\mathrm{V}_{1}^{*}$ and $\mathrm{U}_{2}=\mathrm{V}_{2}^{*}$. In the presence of a magnetic field $B, \mathrm{U}_{1}(B)=\mathrm{V}_{1}^{*}(-B)$ and $\mathrm{U}_{2}(B)=\mathrm{V}_{2}^{*}(-B)$. Similar constraints appear if the sample is a Mesoscopic ring: a ring can be viewed as a black box with incoming and outgoing states connected on two opposite sides of the box. To form a ring, an incoming lead on one side has to be connected to an outgoing lead on the other side. This imposes the constraint $U_{1}=V_{2}$ and $U_{2}=V_{1}$. In general, the unitary transformations determine which universality class, in the random matrix theory language ${ }^{44)}$, the sample belongs to.

\section{MULTITERMINAL CASE AND NOISE CORRELATIONS}

The case where the sample is connected to several leads provides another challenge for the wave packet approach. Here, we will not get into the details of this situation, but rather give the essential ingredients of the approach ${ }^{15)}$. Also, the discussion is restricted to the zero temperature $(\Theta=0)$ case.

We consider a multiterminal sample connected to $p$ leads, and propose to calculate the noise in a given lead $\alpha$. To each lead we associate a chemical potential, and we label each lead with decreasing chemical potential, $\mu_{1}>\ldots>\mu_{\alpha}>\ldots>\mu_{p}$. For $i<\alpha$, in the energy range $\left[\mu_{i}+1, \mu_{i}\right]$, reservoirs 1 to $i$ are injecting electrons in lead $\alpha$. For $i>\alpha$, reservoirs $i+1$ to $p$ inject holes in $\alpha$. In both cases, it is straightforward to determine the transmission matrix associated with each energy interval. In turn, the transmission matrix associated with these intervals yields the eigenvalues (the $T_{i}$ 's) which enter the noise formula. Note that the basic idea of this approach is to reduce the discussion to the two lead case, which we know how to handle. The calculation of the noise in lead $\alpha$ therefore sums up the contribution of each energy interval, and the final result can be expressed in terms of a trace on the block elements of the 
S-matrix:

$$
<\Delta I_{\alpha}^{2}>_{\varepsilon \nu}=\frac{2 e^{2} \delta \nu}{\pi \hbar} \sum_{\eta<\delta}\left(\mu_{\delta}-\mu_{\eta}\right) \operatorname{Tr}\left[\mathbf{s}_{\alpha \delta} \mathbf{s}_{\alpha \delta} \dagger \mathbf{s}_{\alpha \eta} \mathbf{s}_{\alpha \eta} \dagger\right] .
$$

To calculate the noise correlations between two leads $\alpha$ and $\beta$, we consider a fictitious lead $(\alpha+\beta)$. We then proceed as in the previous paragraph to calculate the noise in $(\alpha+\beta)$. The noise in this lead is equal to the noise in $\alpha$, the noise in $\beta$, plus the interference term which represents the correlations between $\alpha$ and $\beta: 2\left\langle\Delta I_{\alpha} \Delta I_{\beta}\right\rangle_{\delta \nu}$. We then obtain, at zero temperature:

$$
<\Delta I_{\alpha} \Delta I_{\beta}>_{\delta \nu}=-\frac{2 e^{2} \delta \nu}{\pi \hbar} \sum_{\eta>\delta}\left(\mu_{\delta}-\mu_{\eta}\right) \operatorname{Tr}\left[\mathbf{s}_{\alpha \eta} \mathbf{s}_{\beta \eta}^{\dagger} \mathbf{s}_{\beta \delta} \mathbf{s}_{\alpha \delta}^{\dagger}\right]
$$

Admittedly, the generalization of the wave packet approach to multiprobe, multiterminal samples requires some thinking, and is so far limited to $\Theta=0$. For $\Theta \neq 0$, Ref. 21 finds it convenient to decompose the noise into equilibrium-like and transport fluctuations: the transport fluctuations bear a negative (positive) sign as in Eq. 15 when the carrier obey the Fermi (Bose) statistics.

\section{NOISE CORRELATIONS IN A Y-SHAPED STRUCTURE}

In 1953, Hanbury-Brown and Twiss performed an experiment ${ }^{48)}$ where two detectors at different locations collected photons emitted from an incoherent light source. The correlations between the two detectors where measured as a function of the distance separating the detectors, and where found to be always positive. This measurement can be viewed as a check that photons are indistinguishable particles which obey Bose-Einstein statistics. Nevertheless, it can also be fully understood in terms of classical electromagnetism ${ }^{49}$ ): the photon bunching effect is a consequence of the superposition principle for light applied to noisy sources.

Fig. 2

It has been suggested ${ }^{15,50}$ that a similar experiment be performed for fermions. A natural "battle field" for this experiment would be to study the noise correlations for electrons injected 
in a Mesoscopic device from one lead, and collected by two other leads: a $Y$ shaped device etched in a GaAs/AlGaAs heterostructure, for example (Fig. 2). We associate the injecting lead with a chemical potential $\mu_{1}$, and fix the chemical potential in lead 2 and 3 at an equal value $\mu_{2}<\mu_{1}$. For simplicity, we also assume $\Theta<<\left|\mu_{1}-\mu_{2}\right|$ so as to ignore thermal effects. Using the results of the previous section, the noise correlations between the collecting leads 2 and 3 is given by:

$$
<\Delta I_{2} \Delta I_{3}>_{\varepsilon}=-\frac{2 e^{2} \delta \nu}{\pi \hbar}\left[\left(\mu_{1}-\mu_{2}\right) \operatorname{Tr}\left[\mathbf{s}_{21}^{\dagger} \mathbf{s}_{21} \mathbf{s}_{31}^{\dagger} \mathbf{s}_{31}\right]\right.
$$

which shows that the correlations are always negative, a direct consequence of the fact that electrons obey Fermi statistics. Ref. 21 also proved that the reverse is true if the carriers are bosons. As the noise correlations measure the likelyhood to detect an electron at a time $t+t^{\prime}$ in lead 3 knowing that an electron was detected in 2 at time $t$, we therefore have a direct analog of the Hanbury- Brown experiment for electrons. In the simplest case, where only one channel propagates in each lead, the effect will be maximal (compared to the noise contributions in each receiving lead) if we construct the Mesoscopic sample so as to minimize the backscattering of the electrons coming from the injecting lead.

\section{EXCHANGE INTERFERENCE IN A FOUR TERMINAL GEOMETRY}

An interesting application of the result for the noise correlations in multiprobe geometries has been pointed out by Büttiker ${ }^{17)}$. For a sample connected to four (or more) probes, Eq. (15) contains products of the block elements of the S-matrix which cannot be written as a matrix multiplied by its hermitian conjugate. Büttiker suggested that this opens the possibility for observing interference effects in a four terminal geometry despite the fact that no coherence exist between different reservoirs.

Fig. 3

Büttiker ${ }^{21)}$ proposes a gedanken experiment in the integer quantum Hall regime where four leads, each containing one edge state, communicate with each other via a quantum dot which 
in this case is a circulating edge state. The leads are labeled from 1 to 4 as one goes around the central island (Fig. 3, taken from Ref. 21). Attention is focused on the noise correlation between leads 2 and 4. In a first step (a) electrons are injected from lead 1: $\mu=\mu_{1}$ and $\mu_{0}=\mu_{2}=\mu_{3}=\mu_{4}\left(\mu_{0}<\mu\right)$, in a second step (b) electrons are injected from lead 3: $\mu=\mu_{3}$ and $\mu_{0}=\mu_{1}=\mu_{2}=\mu_{4}$, and finally (c) electrons are injected from both 1 and $3: \mu=\mu_{1}=\mu_{3}$ and $\mu_{2}=\mu_{4}$. Evaluating the noise correlations in these three cases, Buttiker finds the relation:

$$
\begin{aligned}
<\Delta I_{2} \Delta I_{4}>_{c}= & <\Delta I_{2} \Delta I_{4}>_{a}+<\Delta I_{2} \Delta I_{4}>_{b} \\
& -2 \delta \nu\left(e^{2} / h\right) \int d E\left(f-f_{0}\right)\left[\operatorname{Tr}\left(\mathbf{s}_{21}^{\dagger} \mathbf{s}_{23} \mathbf{s}_{43}^{\dagger} \mathbf{s}_{41}\right)+\operatorname{Tr}\left(\mathbf{s}_{23}^{\dagger} \mathbf{s}_{21} \mathbf{s}_{41}^{\dagger} \mathbf{s}_{43}\right)\right],
\end{aligned}
$$

where $<\Delta I_{2} \Delta I_{4}>_{a, b}$ are the noise correlations associated with steps (a) and (b), which do not contain any phase information, and $f\left(f_{0}\right)$ is the Fermi factor associated with the chemical potential $\mu\left(\mu_{0}\right)$. In contrast to the two first contributions, the last term in Eq. (17) can carry phase information. This is seen by determining the S-matrix associated with this geometry in terms of the transmission/reflection probabilities and the phase shifts associated with the transfer from one reservoir to another. The end result is that:

$$
\operatorname{Tr}\left(\mathbf{s}_{21}^{\dagger} \mathbf{s}_{23} \mathbf{s}_{43}^{\dagger} \mathbf{s}_{41}\right) \propto \exp (i \chi)
$$

where $\chi$ is the total phase accumulated in one cycle around the central island. It would then be possible to observe Aharonov-Bohm type oscillations when measuring noise correlations in the appropriate way. This is quite a surprising result, because unlike in the traditional Aharonov-Bohm effect, here there is no single electron trajectory which traps a magnetic flux. Büttiker ${ }^{17,21)}$ argues that the interference predicted from the noise correlation experiment represents a new type of interference, which arises from exchange effects. In the (different) context of particle physics, a similar phenomenon was pointed out ${ }^{51)} 30$ years ago: the intensity cross correlation between two detectors analyzing the scattering of particles off a target illuminated by two incoherent sources can yield phase information. Indeed, the wave function associated with a system of two particles has to be written as an antisymmetrized product of single particle wave functions. As a consequence, the probability density contains terms which are sensitive to an external flux.

\section{NON STATIONARY AHARONOV-BOHM EFFECT}

A somehow similar effect has been predicted by Lesovik and Levitov ${ }^{26)}$ for a simple, two lead geometry. The sample is bent in the shape of a loop, but the source and drain do not touch each other, so that there is no single electron trajectory which can trap flux. An alternating flux $\phi(t)=\phi_{a} \sin (\Omega t)$ is imposed through the loop. The frequency $\Omega$ is assumed to be small compared to the traversal time of an electron in the sample, and the Fermi energy is assumed to be much larger than the applied bias, as well as the quantum $\hbar \Omega$.

If the field is weak, the essential role played by the magnetic field is to give a phase $\oint$ A.dl to an electron transmitted from the source to the drain. The current predicted in this situation is constant, and has no dependence on flux. 
The central point of this gedanken experiment is that it is possible to observe an oscillatory behavior in the low frequency noise. The derivative of the noise with respect to the applied bias $\partial S / \partial V$ is found to have a staircase-like structure:

$$
\frac{\partial S}{\partial V}=\frac{2 e^{2}}{\pi} \sum_{n} \lambda_{n} \Theta(e V-n \hbar \Omega)
$$

where $\Theta(x)$ is here the Heavyside function, and the height of the steps is given by

$$
\lambda_{n}=T(1-T) J_{n}^{2}\left(\phi_{a} / \phi_{0}\right)
$$

where $T$ is the transmission probability, $\phi_{0}$ is the flux quantum, and $J_{n}$ is the Bessel function of order $n$. By varying the amplitude of the oscillatory flux, the height of the steps oscillates, and decays for large amplitudes. The noise power measurement yields phase information where a simple current measurement fails to do so. The non-stationary Aharonov-Bohm effect is believed to arise from exchange interference between states incident on the sample from the source and the drain, which energies shifted by an amount $\hbar n \Omega$ : the presence of the alternating flux allows these states to "communicate".

\section{NOISE IN CORRELATED ELECTRONS SYSTEMS}

A calculation of noise in the presence of Coulomb repulsion has been proposed by Hershfield ${ }^{22}$. In this work, the Mesoscopic sample consists of two ideal leads (non interacting electrons) connected by a central island where interactions are taken into account. Later on, the central region is taken to be an Anderson impurity site. Also, it is assumed that the tunneling rates on each side of the island are independent of the energy. The calculation is performed using the Keldysh approach, and the lowest order vertex corrections are computed in perturbation theory, using the Hartree approximation. By comparison with the non-interacting case, Hershfield identifies the different contributions. A first term resembles the non interacting result. A second term arises because the electron distribution function at the impurity site is modified by the interaction. A third term is associated with inelastic scattering processes. Finally, the last contribution cannot be identified with corrections to the noninteracting case, and is believed to contain memory effects associated with the interaction: in general, the resulting noise power at low frequencies cannot be written in terms of an effective transmission probability.

Another specific situation allows an effective treatment of the correlations between electrons. Such is the case of Ref. 27, which deals with the non equilibrium noise in the fractional quantum Hall regime for a constriction. The constriction renders the problem one dimensional, and one can then make use of the bosonization methods developed for one dimensional interacting electron systems ${ }^{52)}$ In a constricted quantum Hall geometry, transport is believed to occur primarily at the edges. For a fractional filling factor $\nu=1 / m$, the edge excitations form a single channel chiral Luttinger liquid ${ }^{53}$ [?], characterized by a dimensionless conductance $g=1 / m$. Kane and Fisher ${ }^{27}$ ) The noise in this situation arises from the backscattering of Laughlin quasi particles ${ }^{54}$, which are composed of a fraction $g$ of an electron bound to one 
vortex. As a consequence of this binding, current and voltage fluctuations are locked together at low temperatures.

In the limit of strong backscattering, Fisher and Kane find that the noise reduces to the classical (Poissonian) case. The noise is due to uncorrelated tunneling of electrons through the constriction from source to drain:

$$
S=e \operatorname{coth}\left(\frac{e V_{s d}}{2 k_{B} \Theta}\right)
$$

with $V_{s d}$ the source to drain voltage.

In the opposite limit of weak backscattering, noise arises from the tunneling of Laughlin quasiparticles from one edge to the other. Since the transmission at the constriction is good, this tunneling can also be considered as a Poissonian process. This time, the voltage fluctuations satisfy a classical shot noise form, where the charge of the electron is replaced by the charge of a Laughlin particle ge. Relating the voltage fluctuations to the current fluctuations yields a shot noise formula:

$$
S=g e\left(I_{\max }-<I>\right)
$$

with $I_{\max }=g\left(e^{2} / h\right) V_{s d}$ the current associated with perfect transmission. Because of the prefactor $g e$ in Eq. (22), a noise experiment in this regime would yield a direct observation of fractional charge! In the case of a resonance, where two constrictions isolate a central region ${ }^{27}$ it is possible to suppress the contribution from single quasiparticle tunneling. The dominant process is then the tunneling of pairs of quasiparticles, so that the noise contribution of Eq. (22) has to be multiplied by 2 .

\section{CONCLUSION}

This contribution has attempted to review the work on noise in the last decade, focusing on a few specific topics which were chosen because of their importance in the author's opinion, and/or because they represent a nontrivial generalization of the elastic case. Among the first category is the reduction of shot noise for highly transmissive samples. While this effect has been qualitatively seen in several experiments, it would be useful to repeat these experiments in order to obtain more quantitative results. The role of the statistics of the charge carriers has been emphasized throughout the paper. Noise correlations in a Y-shaped structure and the "new" exchange interference effect were discussed mainly with the goal of motivating an experimental effort in this direction.

The inclusion of interactions ${ }^{22,27)}$ has opened a new direction of research, which will hopefully lead to a better understanding of the constraints imposed by the Coulomb repulsion on the transport of electrons. At this point, it is not clear whether the Coulomb interaction between electrons will further reduce the shot noise power below the level obtained for the single electron result. Our intuition leads us to the belief that this will be the case, simply because in a narrow sample with few transverse channels, the Coulomb interaction will restrict the flow of electrons further than the constraints imposed by the Pauli principle. 
We have omitted a discussion of the calculation of noise at finite frequencies, although there has been some effort in this direction in recent years ${ }^{18)}$. Frankly, we are somewhat uncomfortable with these results because they no not treat the electrons in a self consistent manner. Ideally, when discussing frequency dependent phenomena, one should take into account self inductance effects arising due to the magnetic field created by current flow, as well as capacitive effects ${ }^{55}$ ) associated with the electric fields in the circuitry. Capacitive effects are understood to be at the origin of the Coulomb blockade ${ }^{56}$, [?], but have been vastly neglected in theories of quantum transport at finite frequencies. To be fair, a self consistent treatment of current current correlations at finite frequencies has been proposed recently ${ }^{24}$ but in our opinion, it fails to describe the self consistent screening of electrons at the microscopic level. In conclusion, there are still many issues in this field which need more attention, and the field clearly suffers from a low experiment to theory ratio; this will hopefully change in the near future.

\section{ACKNOWLEDGMENTS}

The viewpoint chosen in this contribution reflects to a large extent a collaboration with $\mathrm{R}$. Landauer, whom I am greatful for stimulating my interest in this topic.

1 I.K. Yanson, A.I. Akimenko and A.B. Verkin, Solid State Commun. 43, 765 (1982); A.I. Akimenko, A.V. Verkin and I.K. Yanson, J. Low Temp. phys. 54, 247 (1984).

2 I.O. Kulik and A.N. Omel'yanchuk, Sov. J. Low Temp. Phys. 10, 158 (1984) (Fiz. Nizk. Temp. 10, 305 (1984)).

3 V. A. Klus, Sov. phys. JETP 661243 (1987) (Zh. EKsp. Teor. Fiz. 93, 2179 (1987)).

4 R. Landauer, Physica D38, 226 (1987).

5 G. B. Lesovik, JETP Lett. 49, 594 (1989).

6 B. Yurke and G. P. Kochlanski, Phys. Rev. B41, 8141 (1990).

7 Y. P. Li, A. Zaslavsky, D. C. Tsui, M. Santos and M. Shayegan, Phys. Rev. B 41, 8388 (1990); Resonant Tunneling in Semiconductors: Physics and Applications, edited by L. L. Chang, E. E. Mendez and C. Tejedor (Plenum, New York 1991).

8 Y. P. Li, D. C. Tsui, J. J. Heremans, J. A. Simmons and G. W. Weimann, Appl. Phys. Lett. 57, 774 (1990).

9 M. Büttiker, Phys. Rev. Lett. 65, 2901 (1990).

10 S. Washburn, R. J. Haug, K. Y. Lee and J. M. Hong, Phys. Rev. B 44, 3875 (1991).

11 A. J. Kil, R. J. J. Zijlstra, M. F. H. Schuurmans and J. P. Andre, Phys. Rev. B41, 5169 (1990).

12 C. W. J. Beenakker and H. van Houten, Phys. Rev. B 43, 12066 (1991).

13 C.W.J. Beenakker and M. Büttiker, Phys. Rev. B 43, 12066 (1991).

14 R. Landauer and Th. Martin, Physica B 175, 167 (1991); 182, 288 (1992).

15 Th. Martin and R. Landauer, Phys. Rev. B 45, 1742 (1992). 
16 G.B. Lesovik and L.S. Levitov, JETP Lett. 58, 230 (1992).

17 M. Büttiker, Phys. Rev. Lett. 68843 (1992).

18 M. Büttiker, Phys. Rev. B 45, 3807 (1992); L.Y. Chen and C.S. Ting Phys. Rev. B 43, 4534 (1991); S.R. Eric Yang, Solid State Commun. 81, 375 (1992).

19 J.W. Wilkins et al., Physica Scripta T42, 115 (1992).

20 A. Shimizu and M. Ueda, Phys. Rev. Lett. 69, 1403 (1992).

21 M. Büttiker, Phys. Rev. B 46, 12485 (1992).

22 S. Hershfield, Phys. Rev. B 46, 7061 (1992).

23 R. Landauer, Phys. Rev. B 47, 16427 (1993).

24 M. Büttiker, A. Prêtre and H. Thomas, Phys. Rev. Lett. 70, 4114 (1993).

25 M.J.M. de Jong and C.W.J. Beenakker, preprint (1993).

26 G. Lesovik and L.S. Levitov, Phys. Rev. Lett. 72, 538 (1994).

27 C.L. Kane and M.P.A. Fisher, Phys. Rev. Lett. 72, 724 (1994).

28 P. Dutta and P. M. Horn, Rev. Mod. Phys. 53, 497 (1981); M. B. Weissman, Rev. Mod. Phys. 60, 537 (1988).

29 S. Feng, P. A. Lee and A. D. Stone, Phys. Rev. Lett. 56, 1960 (1986).

30 J. B. Johnson, Phys. Rev. B 29, 367 (1927); H. Nyquist, Phys. Rev. 32, 110 (1928).

31 J. R. Pierce, Bell Syst. Tech. J. 27, 15 (1948).

32 Van Wees B. J. van Wees et al. Phys. Rev. Lett. 60, 848 (1988).

33 E.A. Montie et al., Nature 350, 594 (1991); Physica B 175, 149 (1991).

34 J.C. Licini et al., Phys. Rev. Lett. 55, 2987 (1985); W.J. Skocpol et al. Phys. Rev. Lett. 56,2865 (1986).

35 S. Washburn and R. Webb, Adv. Phys. 35, 375 (1986).

36 M.P. van Albada, J.F. de Boer and A. Lagendijk, Phys. Rev. Lett. 64, 2787 (1990); J.F. de Boer, M.P. van Albada and A. Lagendijk, Physica B 175, 17 (1991).

37 R. Landauer, Z. Phys. B 68, 217 (1987); M. Büttiker, Y. Imry, R. Landauer and S. Pinhas, Phys. Rev. B 31, 6207 (1985).

38 M. Büttiker, Phys. Rev. Lett. 57, 1761 (1986); M. Büttiker, Phys. Rev. B38, 9375 (1988).

39 L. V. Keldysh, Sov. Phys. JETP 20, 1018 (1965).

40 A. F. Andreev, Sov. Phys. JETP 19, 1228 (1964) (Zh. Eksp. Teor. Fiz. 46, 1823 (1964)).

41 M. Büttiker and R. Landauer, Phys. Rev. Lett. 49, 1739 (1982).

42 S. Hershfield, Phys. Rev. Lett. 70, 2134 (1993).

43 M. Büttiker and Th. Martin, unpublished (1991).

44 P. A. Mello, P. Peyreyra and N. Kumar, Ann. Phys. 181, 290 (1988); P. A. Mello and J. L. Pichard, J. Phys. I 1, 493 (1991). 
45 J. K. Jain and S. A. Kivelson, Phys. Rev. Lett. 60, 1542 (1988), and Phys. Rev. B 37, 4276 (1988); P. Streda, J. Kucera and A. H. MacDonald, Phys. Rev. Lett. 59, 1973 (1987).

46 S. Washburn, A. B. Fowler, H. Schmid and D. Kern, Phys. Rev. Lett. 61, 2801 (1988); R. J. Haug, A. H. MacDonald, P. Streda and K. v. Klitzing, Phys. Rev. Lett. 61, 2797 (1988).

47 Th. Martin and S. Feng, Phys. Rev. Lett. 64, 1971 (1990).

48 R. Hanbury Brown and R. Q. Twiss, Nature 177, 27 (1956); R. Hanbury Brown and R. Q. Twiss, Proc. Royal. Soc. London Ser. A 242, 300 (1957); ibid. 243, 291 (1957).

49 E.M. Purcell, Nature 178, 1449 (1956).

50 S. Murphy, private communication.

51 M. 1. Goldberger, H.W. Lewis, and K.M. Watson, Phys. Rev. 132, 2764 (1963).

52 V. J. Emery, in Highly Conducting One Dimensional Solids, J. T. Devreese, R. P. Evrard and V. E. van Doren eds. (Plenum, New York, 1979), p.327; J. Solyom, Adv. Phys. 28, 201 (1979).

53 X.G. Wen, Phys. Rev. B 43, 11025 (1991); Phys. Rev. Lett. 64, 2206 (1990).

54 R.B. Laughlin, Phys. Rev. Lett. 50, 1395 (1983).

55 R. Landauer, Phisica Scripta T 42, 100 (1992).

56 see for example: C.W.J. Beenakker and H. van Houten, in Single Charge Tunneling (eds. H. Grabert and M.H. Devoret), Plenum, New York (1991).

\section{DISCLAIMER}

This report was prepared as an account of work sponsored by an agency of the United States Government. Neither the United States Government nor any agency thereof, nor any of their employees, makes any warranty, express or implied, or assumes any legal liability or responsibility for the accuracy, completeness, or usefulness of any information, apparatus, product, or process disclosed, or represents that its use would not infringe privately owned rights. Reference herein to any specific commercial product, process, or service by trade name, trademark, manufacturer, or otherwise does not necessarily constitute or imply its endorsement, recommendation, or favoring by the United States Government or any agency thereof. The views and opinions of authors expressed herein do not necessarily state or reflect those of the United States Government or any agency thereof. 